Abstract 82 Table 1 Characteristics of patients with either incident heart failure, incident loop diuretics or both.Data are median and interquartile range (IQR) or $\mathrm{n}(\%)$

\begin{tabular}{|l|c|c|c|}
\hline & $\begin{array}{c}\text { Loop Diuretics Only } \\
(\mathrm{n}=41,570)\end{array}$ & $\begin{array}{c}\text { Heart Failure Only } \\
(\mathrm{n}=5,925)\end{array}$ & $\begin{array}{c}\text { Both } \\
(\mathrm{n}=12,134)\end{array}$ \\
\hline Age (years) & $74(19)$ & $71(21)$ & $78(16)$ \\
\hline Sex- women & $27,585(66 \%)$ & $2,366(40 \%)$ & $6,493(54 \%)$ \\
\hline Ethnicity & & & \\
\hline White & $21,460(52 \%)$ & $3,298(56 \%)$ & $7,175(59 \%)$ \\
\hline Missing & $19,687(47 \%)$ & $2,525(43 \%)$ & $4,782(39 \%)$ \\
\hline Died within 3 years & $11,294(27 \%)$ & $2,220(37 \%)$ & $5,769(48 \%)$ \\
\hline$\%$ of Women & $6,562(24 \%)$ & $1,095(46 \%)$ & $3,232(50 \%)$ \\
\hline$\%$ of Men & $4,732(34 \%)$ & $1,125(32 \%)$ & $2,537(45 \%)$ \\
\hline
\end{tabular}

\section{CHARACTERISTICS AND OUTCOMES OF PATIENTS WITH SUSPECTED HEART FAILURE AND ELEVATED NATRIURETIC PEPTIDES REFERRED TO A NICE-COMPLIANT HEART FAILURE CLINIC}

Alice Zheng, Elena Cowan, Legate Philip, Kaushik Guha, Paul R Kalra, Geraint Morton. Portsmouth Hospitals NHS Trust

\subsection{6/heartjnl-2019-BCS.81}

Introduction Chronic heart failure (HF) represents a substantial and growing public health and financial burden and has a poor prognosis. National Institute for Health and Care Excellence (NICE) guidelines and quality standards recommend timely specialist assessment for patients with suspected HF and raised natriuretic peptides. However, data on the outpatient population assessed, diagnostic rates of HF and outcomes for patients on this pathway are not known. This is in contrast to patients hospitalised with acute HF who are well described in the UK. Our aim is to describe real world data from a large patient cohort with suspected HF investigated according to NICE protocols.

Methods All patients with suspected HF and elevated NTproBNP referred to a dedicated HF clinic between January 2014 - December 2016 were included. Patients underwent specialist assessment, echocardiography and diagnosis in a onestop clinic within 2 weeks (NTproBNP $>2000 \mathrm{pg} / \mathrm{ml}$ ) or 6 weeks (NTproBNP 400-2000pg/ml) in accordance with NICE guidelines.

Patient baseline characteristics and co-morbidities were recorded prospectively. 1 year all-cause hospitalisation (1 or more admissions) and mortality rates were retrospectively calculated from the date of NTproBNP measurement using hospital coding data and electronic patient records.

Outcomes were compared between those referred on the 2 and 6 week pathways; and between those diagnosed with Heart Failure (HF) vs. Not Heart Failure (NHF) after specialist assessment.

Results Out of 1042 consecutive patients referred, 1013 had NTproBNP measured and were included. 544 (54\%) were on the 2-week and 469 (46\%) on the 6-week pathway. 543 (54\%) were diagnosed with HF; 300 (55\%) with Heart Failure with reduced Ejection Fraction (HFrEF) and 243 (45\%) with Heart Failure with preserved Ejection Fraction (HFpEF). $454(45 \%)$ were diagnosed as NHF.

A diagnosis of $\mathrm{HF}$ was made in $383(70 \%)$ in the 2-week and $160(34 \%)$ in the 6 -week pathway $(\mathrm{p}<0.001)$. Of those with HF, HFrEF was diagnosed in $236(62 \%)$ in the 2-week and $64(40 \%)$ in the 6 -week pathway $(\mathrm{p}<0.001)$. Patients characteristics comparing the 2 and 6-week pathways and the HF and NHF groups are shown in tables $1 \& 2$.

For the entire population the 1-year hospitalisation rate was 324/1013 (32\%) and mortality 112/1013 (11\%).

There were significantly higher rates of both mortality (88 [16\%] vs 24 [5\%] p <0.001) and hospitalisation (199 [37\%] vs. $125[27 \%] \mathrm{p}=0.001)$ in the 2 -week compared to the 6week pathway. There was a significantly higher rate of hospitalisation in the HF group compared to the NHF group (189 [35\%] vs 123 [27\%]) $\mathrm{p}=0.009)$ but no difference in mortality (64 [12\%] vs 39 [9\%] $\mathrm{p}=0.099$ ). Figures $1 \& 2$.

Conclusion The prognosis is relatively poor for outpatients with suspected chronic HF and raised natriuretic peptides, with high rates of adverse outcomes observed despite specialist investigation in accordance within NICE timeframes. This is regardless of the final diagnosis, as mortality is comparable between HF and NHF groups.

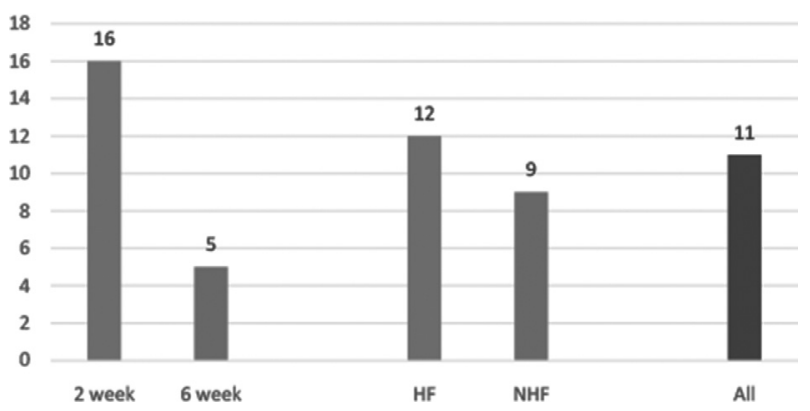

Abstract 83 Figure 11 year All-cause mortality (\%) comparing 2 vs. 6 -week pathways and HF vs. NHF groups

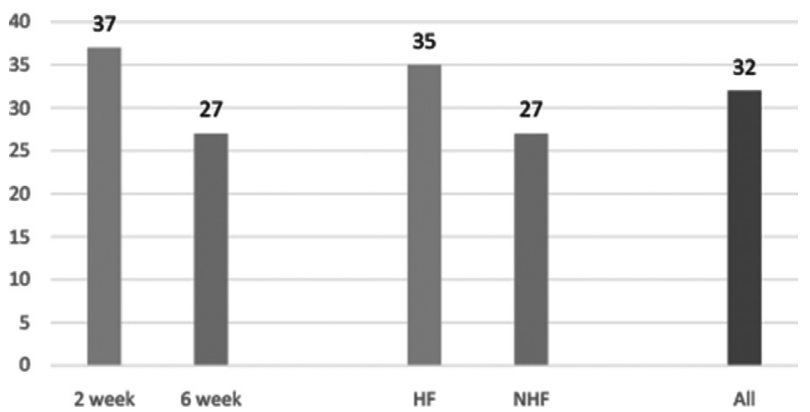

Abstract 83 Figure 21 year All-cause hospitalisation (\%) comparing 2 vs. 6 -week pathways and HF vs. NHF groups 